\title{
Determinación de compuestos polares por TLC-FID en aceites refinado y semi-hidrogenado de soja sometidos a calentamiento prolongado
}

\author{
Por J. L. Cruzian, A. J. Inhamuns y D. Barrera-Arellano \\ Lab. Óleos e Gorduras, FEA/UNICAMP, Caixa Postal 6091, Campinas, S. P. 13.081-970, Brasil. \\ Tel: 55(19) 78884 23, Fax: 55(19)2 391186 E-mail: barrera@ ccsun.unicamp.br
}

\section{RESUMEN}

Determinación de compuestos polares por TLC-FID en aceites refinado y semi-hidrogenado de soja sometidos a calentamiento prolongado.

La degradación térmica de aceites y grasas lleva a la formación de compuestos polares, motivo por el cual su determinación es usada como control de calidad de aceites y grasas utilizadas en fritura. Muestras de aceite de soja refinado y semi-hidrogenado fueron calentadas durante 30 y 60 horas respectivamente y el contenido de compuestos polares fue determinado utilizando el método oficial IUPAC-AOAC y TLCFID. Las muestras de aceite y las fracciones separadas en la columna fueron aplicadas en los "chromarods" y desarrolladas en éter de petróleo: éter etílico $(93: 7 \mathrm{v} / \mathrm{v})$. La determinación de compuestos polares por el método IUPAC y por TLC-FID, dieron resultados similares $(P$-value $<<0,001)$, aunque los obtenidos por el segundo método fueron superiores, cuando los compuestos polares superan el $16 \%$. Los resultados obtenidos indican que el estado de descomposición de aceites y grasas medido por la cantidad de compuestos polares puede ser determinado alternativamente por TLC-FID, presentando una significativa reducción en el tiempo, mano de obra y volumen de disolventes.

PALABRAS-CLAVE: Aceite de soja refinado - Aceite de soja semi-hidrogenado - Compuestos polares - latroscan - TLC/FID.

\section{SUMMARY}

Determination of polar compounds by TLC-FID in refined and partially hidrogenated soja heated oils.

The thermal degradation of oils and fats leads to the formation of polar compounds, which is the reason why their determination is adopted for the quality control of the oil and fat used in frying. Samples of refined and partially hydrogenated soybean oil were heated for 30 and 60 hours respectively, and the polar compounds content were determined using the IUPAC-AOAC official method and TLC-FID. The samples of oil and the fractions separated on the column were applied to the chromarods and developed in petroleum ether: diethyl ether $(93: 7 \mathrm{v} / \mathrm{v})$. The determination of polar compounds by IUPAC method and by TLC-FID presented similar results ( $P$-value $<<0,001)$ although when the content was greater than $16 \%$ the second method gave higher values. The results showed that the state of degradation of oils and fats as measured for the quantity of polar compounds can be determined alternatively by TLC-FID, presenting a significant reduction in time, hand work and solvent volume.

KEY-WORDS: latroscan - Partially hidrogenated soja oil Polar compounds - Refined soja oil - TLC/FID.

\section{INTRODUCCIÓN}

Durante el proceso de fritura, los aceites y grasas son sometidos a grandes períodos de calentamiento expuestos al aire. En estas condiciones, tienen lugar cambios físicos y químicos que originan alteraciones en el flavor, olor, color y valor nutricional.

En el proceso están incluidos oxígeno molecular, ácidos grasos insaturados, triglicéridos, así como los productos de la hidrólisis de triglicéridos que puede reaccionar para formar polímeros y otros productos. En suma, muchas de las reacciones se interrelacionan y se forma una mezcla compleja de productos (Cuesta, et al., 1993; Márquez-Ruiz, et al., 1995).

Debido a las consecuencias toxicológicas y nutricionales (Clark y Serbia, 1991; Taylor et al., 1983), el nivel de alteración de los aceites y grasas durante el proceso de fritura debe ser determinado. Numerosos métodos han sido propuestos para definir este nivel de degradación (Melton, et al., 1994; Shahidi, 1995; Warner y Frankel, 1985; White, 1991) dentro de los cuales la cantidad de compuestos polares (Dobarganes, et al., 1989) ha sido incluido como indicador de calidad de grasas de fritura en la legislación de algunos países, como España (1989), que establecen como valor máximo $25 \%$ de compuestos polares para el descarte de estos productos.

En el método oficial de IUPAC/AOCS para la determinación de los compuestos polares se utiliza la cromatografía en columna clásica con sílice $5 \%$ hidratada (IUPAC, 1987). Este procedimiento hace la separación del aceite en dos fracciones, una apolar constituida de triglicéridos y una polar compuesta de triglicéridos 
oxidados y polimerizados. A pesar de este método dar una excelente indicación del estado de degradación de los aceites y grasas durante el calentamiento, no separa totalmente los componentes alterados de las moléculas sin carga. Por ejemplo, monómeros de ácidos grasos cíclicos, eluyen tanto en la fracción polar cuanto en la apolar (Sebedio, et al., 1986), además este método consume grandes cantidades de disolventes y es bastante laborioso.

La técnica de cromatografía en capa fina y detección por ionización en llama (TLC-FID) viene siendo usada intensivamente en el análisis cualitativo y cuantitativo de lípidos (Coderch, et al., 1995). Sebedio, et al., (1987), cuantificaron por TLC-FID los compuestos polares, en aceites utilizados en restaurantes, comparando este método con el oficial IUPAC (1987) y el de cartuchos SEP-PACK (Sebedio, et al., 1986).

El objetivo del presente estudio fue separar y cuantificar por TLC-FID la fracción polar de aceites de soja, refinado y semi-hidrogenado, formada durante el calentamiento prolongado de los mismos, evaluando su precisión y conveniencia de utilización.

\section{MATERIAL Y MÉTODOS}

Aceites comerciales de soja, refinado y semihidrogenado (Cargill Agrícola, S.A.; Mairinque, S.P., Brasil; Sadia, SP., Brasil), fueron sometidos a un prolongado período de calentamiento continuo a $180^{\circ} \mathrm{C}$ $\pm 5^{\circ} \mathrm{C}$, durante el cual se retiraron muestras a intervalos de tiempo regulares. El aceite semi-hidrogenado fue calentado por 60 horas, colectando 16 muestras, mientras el aceite refinado fue por 30 horas y 14 muestras. En las muestras originales fue determinado su porcentaje de ácidos grasos libres (método Ca $5 \mathrm{a}$ 40 AOCS, 1993) y el índice de peróxido (Cd 20-91 AOCS, 1993).

\section{Separación y cuantificación de las fracciones polar y apolar mediante cromatografía en columna de sílice (IUPAC/AOAC, 1987)}

Todas las muestras fueron determinadas por duplicado en silicagel (Merck 7734, 0,063-0,20 mm) hidratada $5 \%$. La separación de la fracción polar fue hecha con $200 \mathrm{~mL}$ de éter de petróleo/éter etílico 87:13 (v/v). La pureza de cada fracción fue confirmada por TLC y por TLC-FID. Las placas de silicagel (Merck 7747; 0,25 $\mathrm{mm}$ ) fueron desarrolladas en una mezcla éter de petróleo/éter etílico/ácido acético 70:30:1 (v/v/v) y reveladas con una solución de $10 \%$ de ácido fosfomolíbdico en etanol. En los análisis de las fracciones por TLC-FID, las condiciones cromatográficas fueron iguales a las usadas en las muestras de aceites.

Los compuestos polares fueron calculados de dos maneras: como indica la norma, a partir de la primera fracción eluída (apolares), donde el valor de porcentaje de compuestos polares fue obtenido por diferencia de 100, y segundo, a partir de las dos fracciones eluídas, donde el porcentaje de compuestos polares (segunda fracción) fue calculada con relación al peso total de la muestra recuperada.

\section{Separación y cuantificación de las fracciones polar y apolar mediante TLC-FID}

Las muestras recogidas en diferentes tiempos de calentamiento fueron preparadas en concentraciones de $10 ; 8$ y $5 \mu \mathrm{g} / \mu \mathrm{L}$ en tolueno aplicándose $1 \mu \mathrm{L}$ de muestra en chromarods S-III por triplicado. A continuación, se utilizó el método para TLC-FID propuesto por Sebedio et al. (1987) con las siguientes modificaciones: 1) Se eliminó el ácido acético del solvente de elución, probando los sistemas constituidos de éter de petróleo/éter etílico 80:20 y 87:13 (v/v) durante 30 minutos y 93:7 (v/v), durante 22 minutos y, posteriormente, secados a $50{ }^{\circ} \mathrm{C}$ durante 10 minutos, y 2) No se utilizó el patrón interno de metil heptadecanoato. Finalmente fueron evaluados $(0,32 \mathrm{~cm} / \mathrm{s})$ en el detector de ionización de llama (FID) del IATROSCAN MK-5 (latron Lab. Inc. Tokyo, Japan).

El factor de respuesta (Fr) del detector FID fue calculado a partir de las fracciones polar y apolar, separadas en la columna (método IUPAC/AOAC). Para ello, soluciones de los compuestos polares y apolares en igual cantidad $(11 ; 7,7$ y $3,0 \mu \mathrm{g} / \mu \mathrm{L})$ fueron aplicadas en los chromarods, desarrollados en éter de petróleo/éter etílico 93:7 (v/v) y evaluadas en las condiciones cromatográficas citadas anteriormente.

\section{Análisis estadístico}

El análisis de variancia fue el modelo adoptado para comparar los valores de los compuestos polares entre sí (IUPAC/AOAC e TLC-FID), de aceite refinado y semi-hidrogenado, considerando la variación de temperatura, a un nivel de significación del $1 \%$.

\section{RESULTADOS Y DISCUSIÓN}

Las muestras originales de aceite de soja refinado y semi-hidrogenado presentaron un porcentaje de ácidos grasos libres de 0,20 y 0,15, respectivamente y valores de índice de peróxido de 0,97 y 0,74.

\section{Determinación de compuestos polares (CP) por cromatografía en columna y por TLC-FID}

Los resultados obtenidos en la cuantificación de CP a través del método oficial (IUPAC/AOAC) (método I) y por TLC-FID (método II), están relacionados en la 
Tabla I

Compuestos polares (\%) en aceite de soja, refinado y semi-hidrogenado, calentado a $180{ }^{\circ} \mathrm{C}$ a diferentes tiempos. Cuantificación por los Métodos IUPAC/AOAC y TLC-FID

\begin{tabular}{|c|c|c|c|c|c|c|c|c|}
\hline \multirow[b]{3}{*}{$T \Delta$ (h) } & \multicolumn{4}{|c|}{ Aceite refinado } & \multicolumn{4}{|c|}{ Aceite semi-hidrogenado } \\
\hline & \multicolumn{3}{|c|}{ Método I (IUPAC/AOAC) } & \multirow{2}{*}{$\frac{\text { Mét. II }}{\text { TLC-FID }}$} & \multicolumn{3}{|c|}{ Método I (IUPAC/AOAC) } & \multirow{2}{*}{$\frac{\text { Mét. II }}{\text { TLC-FID }}$} \\
\hline & A & B & C & & A & B & C & \\
\hline 0 & 98.34 & 4.66 & 6.25 & 6.24 & 98.25 & 3.54 & 4.68 & 2.87 \\
\hline 2 & 98.57 & 6.77 & 8.07 & 7.28 & - & - & - & - \\
\hline 4 & 98.56 & 8.07 & 9.39 & 9.58 & 95.47 & 3.90 & 5.75 & 4.48 \\
\hline 6 & 99.44 & 9.90 & 10.40 & 11.05 & - & - & - & - \\
\hline 8 & 97.47 & 11.40 & 11.80 & 12.54 & 97.68 & 5.34 & 7.06 & 6.16 \\
\hline 10 & 99.21 & 12.25 & 12.95 & 13.62 & - & - & - & - \\
\hline 12 & 98.06 & 14.55 & 16.00 & 16.92 & 98.31 & 6.20 & 7.82 & 7.20 \\
\hline 14 & 99.50 & 16.49 & 17.27 & 20.03 & - & - & - & - \\
\hline 16 & - & - & - & - & 98.84 & 7.16 & 8.60 & 8.47 \\
\hline 18 & 98.18 & 19.60 & 21.06 & 23.07 & - & - & - & - \\
\hline 20 & - & - & - & - & 97.69 & 9.02 & 10.96 & 11.11 \\
\hline 22 & 98.87 & 23.23 & 24.10 & 27.96 & - & - & - & - \\
\hline 24 & 98.39 & 25.03 & 26.37 & 29.80 & 96.77 & 11.04 & 13.24 & 13.46 \\
\hline 26 & 97.76 & 27.58 & 29.19 & 30.52 & - & - & - & - \\
\hline 28 & 97.67 & 30.26 & 31.89 & 33.07 & 98.77 & 12.48 & 14.17 & 15.00 \\
\hline 30 & 97.36 & 33.16 & 34.66 & 40.30 & - & - & - & - \\
\hline 32 & - & - & - & - & 98.01 & 13.66 & 15.37 & 16.91 \\
\hline 36 & - & - & - & - & 97.77 & 14.90 & 16.77 & 18.00 \\
\hline 40 & - & - & - & - & 99.04 & 16.79 & 17.60 & 19.51 \\
\hline 44 & - & - & - & - & 98.22 & 17.66 & 18.24 & 21.80 \\
\hline 48 & - & - & - & - & 98.59 & 18.75 & 20.24 & 22.08 \\
\hline 52 & - & - & - & - & 98.70 & 20.27 & 22.64 & 23.60 \\
\hline 56 & - & - & - & - & 99.02 & 22.07 & 23.25 & 26.03 \\
\hline 60 & - & - & - & - & 97.66 & 25.20 & 26.95 & 28.01 \\
\hline
\end{tabular}

$\mathrm{T} \Delta$ - Tiempo de calentamiento en horas.

A - Recuperación (\%) de la muestra fraccionada en la columna cromatográfica $R(\%)=\frac{m p+m a}{m} \times 100$

B - Compuestos polares (\%) extraídos, considerando el $\%$ de recuperación $C p(\%)=\frac{m p}{m} \times A$

C - Compuestos polares (\%) obtenidos por diferencia de los compuestos apolares $C p(\%)=\frac{m-m a}{m} \times 100$

$\mathrm{m}$ - Peso inicial de muestra $(\mathrm{g})$

$\mathrm{mp}$ - Peso de la fracción polar separada $(\mathrm{g})$

$\mathrm{ma}$ - Peso de la fracción apolar separada $(\mathrm{g})$

Tabla I. Partiendo del fraccionamiento de las muestras en la columna cromatográfica, el porcentaje de compuestos polares fue cuantificado de varias maneras como se muestra en la leyenda de la tabla I. El valor de CP de la columna $\mathrm{C}$ (Tabla I), fue calculado siguiendo el procedimiento oficial de la IUPAC. El número de muestras que superaron el límite de $25 \%$ de $C P$, fue de 5 (con más de 20 horas de calentamiento) y 2 (con más de 53 horas) para aceite refinado y semi-hidrogenado, respectivamente. El aceite semi-hidrogenado, que posee una estabilidad oxidativa mayor, fue calentado por un tiempo mayor, para obtener un nivel de alteración similar al del aceite refinado.
Los valores para compuestos polares, obtenidos por los métodos TLC-FID y IUPAC en este estudio, son muy similares durante todo el proceso de degradación térmica. Sebedio et al., (1987) cuando compararon estos métodos encontraron valores diferentes, principalmente cuando el cálculo era hecho con la ecuación de regresión de los compuestos polares determinados por TLC-FID, atribuyendo esta variación a la composición de la fracción polar, diferente para cada tipo de aceite y del tratamiento térmico al cual es sometido. 


\section{Estudio del efecto del solvente en la elución de las muestras en TLC-FID}

En la figura 1 se muestran los cromatogramas obtenidos con los diferentes sistemas disolventes, donde puede observarse que, para los objetivos de este trabajo, la mezcla de éter de petróleo/éter etílico 93:7 es la más adecuada, ya que se obtuvieron sólo dos picos (1c), correspondiendo a las fracciones polar y apolar obtenidas en la cromatografía de columna.
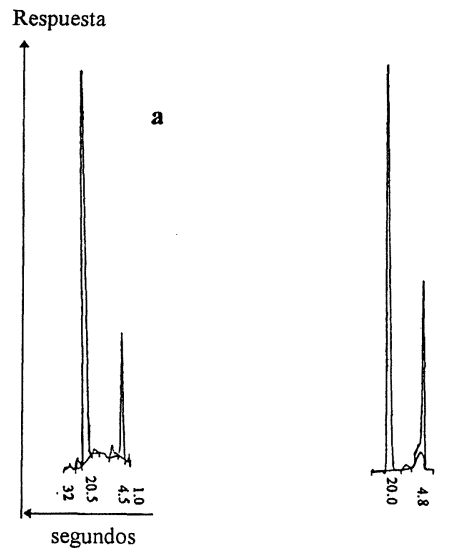

b

Figura 1
Cromatogramas TLC-FID de aceite semi-hidrogenado con 60 horas de calentamiento, en éter de petróleo: éter etílico 80:20 (a); 87:13 (b) y 93:7 (c).

La figura 2 muestra los cromatogramas del aceite refinado (I) y semihidrogenado (II) en diferentes tiempos de calentamiento, observándose un aumento de la fracción polar a medida que aumenta el tiempo de calentamiento y cuyos datos completos son presentados en la tabla $\mathrm{l}$.

\section{Efecto de la concentración de la muestra en la respuesta del detector}

La concentración de $10 \mu \mathrm{g} / \mu \mathrm{L}$ presentó problemas de detección en las muestras con porcentajes elevados de compuestos apolares, debido a la saturación del detector. Por otro lado, con la concentración de 5 $\mu \mathrm{g} / \mu \mathrm{L}$ de muestra, la cuantificación de los compuestos polares, cuando se encuentran en bajas concentraciones, necesitó un aumento en la sensibilidad, originando cromatogramas menos reproducibles. La concentración que presentó los mejores resultados, a bajas y elevadas concentraciones de polares y apolares, fue la de $8 \mu \mathrm{g} / \mu \mathrm{L}$.

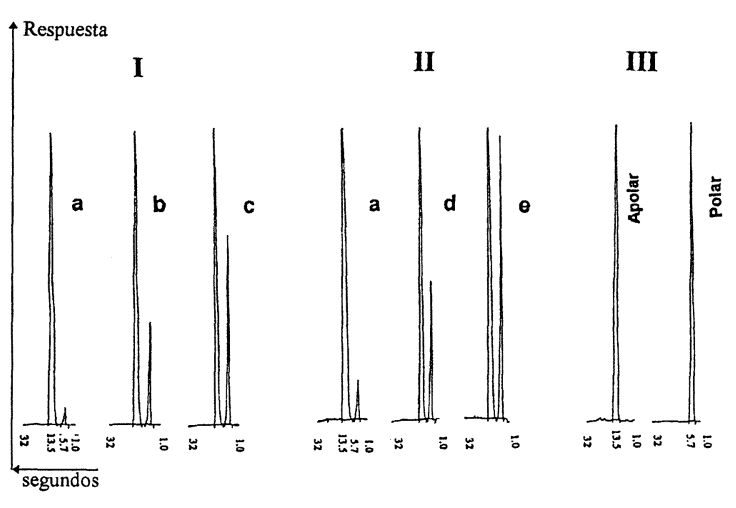

Figura 2

Cromatogramas TLC-FIC de aceite semi-hidrogenado (I) y refinado (II) de soja, calentado en diferentes tiempos: a) 0;

b) 32 ; c) 56 ; d) 14 y e) 28 horas, y de las fracciones (III) obtenidas por cromatografía de columna (Método IUPAC/AOAC). Sistema de disolvente éter de petróleo: éter etílico (93:7).

\section{Evaluación de la pureza de las fracciones separadas en columna cromatográfica por TLC-FID}

El análisis por TLC-FID de las fracciones polares y apolares obtenidas del fraccionamiento de las muestras en silicagel, mostró que la contaminación entre fracciones es muy baja, siendo que pocos puntos llegaron a $4 \%$. Además se observó que una pequeña cantidad de compuestos polares siempre eluye con mayor frecuencia y proporcionalmente, junto con los CA. Tal resultado, probablemente, refleja la diferencia constatada, en muestras con $\mathrm{CP}$ menores que $10 \%$ entre los métodos IUPAC/AOAC (C) y TLC-FID (Tab. I Fig. 4).

\section{Factor de respuesta (Fr) del detector de ionización de llama (FID)}

Después de verificar la pureza por TLC, se aplicaron las fracciones apolar y polar obtenidas por el método IUPAC/AOAC en cantidades iguales a diferentes concentraciones (3; 7,7 y $11 \mu \mathrm{g} / \mu \mathrm{L})$, observando que el porcentaje de área obtenido para los compuestos apolares varía entre 48 y $52 \%$ y viceversa para los polares (Fig. 3). Se concluye, por tanto, que para el conjunto de compuestos polares y apolares la respuesta del detector es similar, por lo que no hay necesidad del uso de factor de corrección en esta determinación. 


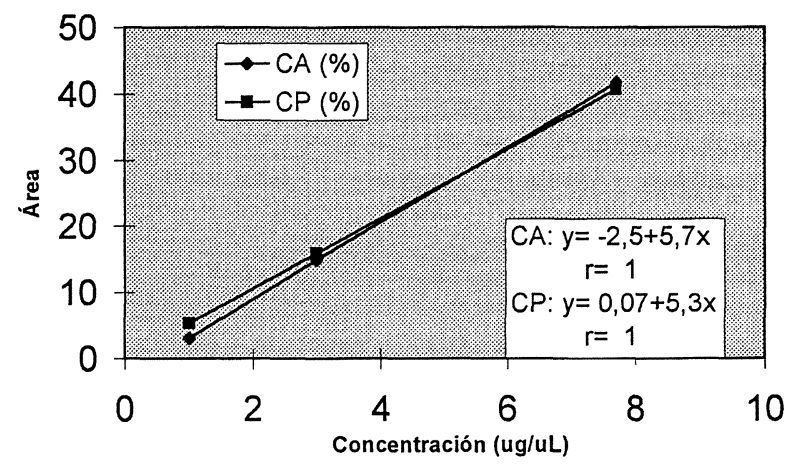

Figura 3

Factor de respuesta del detector FID para compuestos polares y apolares aplicados en concentraciones iguales.

\section{Estudio comparativo entre los métodos}

Las determinaciones de CP por los dos métodos presentaron comportamientos similares durante el tiempo de calentamiento, independiente del tipo de muestra analizada, como puede ser observado en la figura 4.

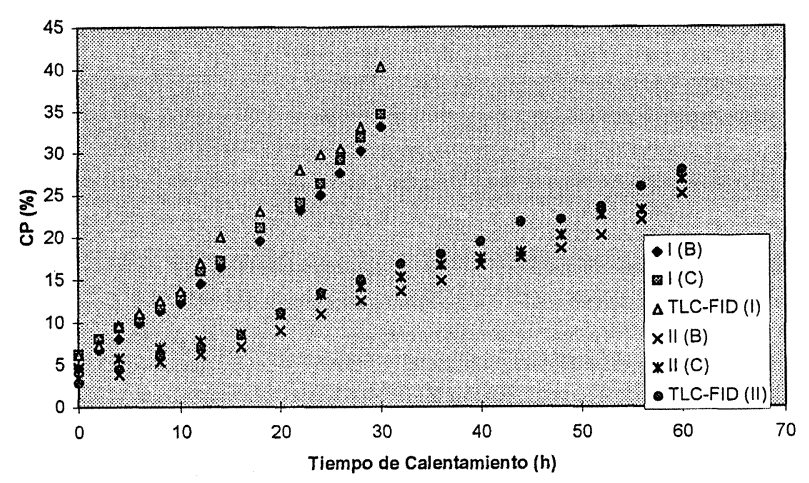

Figura 4

Compuestos polares de aceite refinado (I) y semi-hidrogenado (II), obtenidos por el método oficial (IUPAC/AOAC) y por TLC-FID.
En la comparación de los resultados fue usada la metodología de Análisis de Variancia, donde la temperatura de calentamiento fue considerada como factor en el modelo adoptado. Esto se debe al hecho de que fue observado una variación en las respuestas para $\mathrm{CP}$ en intervalos de clases diferenciadas, cuando los métodos IUPAC(C) y TLC-FID son comparados. En la figura 4, las curvas para los dos tipos de muestras se cruzan en determinado punto (aproximadamente 10\% de $(\mathrm{P})$. Hasta este punto de intersección, valores mayores fueron encontrados en el método oficial. Entre 9 y 16\%, resultados muy próximos fueron obtenidos por los dos métodos. Para valores mayores que $16 \%$ de $C P$, una diferencia mayor y más acentuada que las anteriores, fue observada para los análisis por TLC-FID. La comparación entre los datos obtenidos por el método oficial ( $B$ y $C$ ) y por TLC-FID, no mostró diferencia significativa a nivel de $1 \%(P$-value $<<0,001)$, en cualquiera de los intervalos.

Las ecuaciones de regresión lineal $(y)$, coeficiente de correlación ( $r$ ) y desviación estándar (sd) para las curvas de la fracción polar, obtenidas por los métodos IUPAC/AOAC y TLC-FID, están en la tabla II. Las diferencias entre los valores encontrados por TLC-FID para el aceite refinado y semi-hidrogenado, pueden ser justificadas en función de que para el aceite refinado, los valores de CP obtenidos después de 30 horas de calentamiento están fuera de la zona lineal de la curva. Así, si la regresión lineal para el aceite refinado fuera calculada con la exclusión del último punto (30 horas), los nuevos valores serían $\mathrm{Sd}=0,76$; $r=0,997$ e $y=5,28+0,99 x$ más próximos de los obtenidos para el semi-hidrogenado.

Los resultados obtenidos muestran que la determinación de compuestos polares en aceites puede ser hecha con seguridad utilizando la técnica TLC-FID.

A pesar de que el método de la IUPAC (1987) es simple, reproducible y tiene una buena correlación con métodos más precisos de determinación de la degradación de aceites (White, 1991), se trata de un método que presenta como desventajas: el alto consumo de disolventes, el largo tiempo de análisis y el uso intensivo de mano de obra, factores que en caso de utilizar el método TLC-FID propuesto, son minimiza-

Tabla II

Ecuación de regresión linear (y), desviación standard (Sd) y coeficiente de correlación (r) para la determinación de compuestos polares (CP) por los métodos IUPAC/AOAC y TLC-FID

\begin{tabular}{|c|c|c|c|c|c|c|}
\hline \multirow[t]{2}{*}{ Método } & \multicolumn{3}{|c|}{ Aceite refinado } & \multicolumn{3}{|c|}{ Aceite semi-hidrogenado } \\
\hline & Ecuación & Sd & $\mathbf{r}$ & Ecuación & Sd & $\mathbf{r}$ \\
\hline IUPAC/AOAC (B) & $Y=4.13+0.91 x$ & 0.84 & 0.996 & $Y=2.37+0.35 x$ & 0.65 & 0.996 \\
\hline IUPAC/AOAC (C) & $Y=5.09+0.92 x$ & 1.05 & 0.994 & $Y=4.03+0.35 x$ & 0.78 & 0.993 \\
\hline TLC-FID & $Y=4.77+1.05 X$ & 1.48 & 0.991 & $Y=2.79+0.42 x$ & 0.57 & 0.997 \\
\hline
\end{tabular}

B - Compuestos polares (\%), considerando el \% de recuperación de la columna.

$\mathrm{C}$ - Compuestos polares (\%) por diferencia del peso inicial. 
dos, principalmente en lo que se refiere a los disolventes que tienen que ser descartados al final de la determinación. En este caso la reducción de volumen de disolventes es del orden de 1:10 cuando se compara con IUPAC para una muestra, además por TLCFID pueden ser determinadas hasta 10 muestras con el mismo disolvente.

Por lo antes expuesto se puede concluir que la técnica TLC-FID es viable y precisa para ser utilizada en la determinación de la degradación de aceites y grasas sometidas a procesos térmicos.

\section{BIBLIOGRAFÍA}

Clark, W. L., Serbia, G. W. (1991). - «Safety aspects of frying fats and oils".- Food Technol. 45, 84-89.

Coderch, L., De la Maza, A., Soriano, C., Erra, P., Parra, J. L. (1995). - "Chromatographic characterization of internal polar lipids from wool».- J. Am. Oil Chem. Soc. 72, 715720.

Cuesta, C., Sánchez-Muniz, F. J., Garrido-Polonio, C., LópezVarela, S., Arroyo, R. (1993). - «Thermoxidative and hydrolytic changes in sunflower oil used in fryings with a fast turnover of fresh oil».- J. Am. Oil Chem. Soc. 70, 1069-1073.

Dobarganes, M.C., Pérez-Camino, M. C., Márquez-Ruiz, G. (1989). - «Determinación de compuestos polares en aceites y grasas de fritura».- Grasas y Aceites 40, 35-38.

España. -Boletín Oficial del Estado, núm. 26 del 31 de enero de 1989. Orden 2265. - «Norma de calidad para los aceites y grasas calentados".-

Márquez-Ruiz, G., Tasioula-Margari, M., Dobarganes, M. C. (1995). - «Quantitation and distribution of altered fatty acids in frying fats".- J. Am. Oil Chem. Soc. 72, 1171. 1176.
Melton, S. L., Jafar, S., Sykes, D., Trigiano, M. K. (1994). - "Review of stability measurements for frying oils and fried food flavor".- J. Am. Oil Chem. Soc. 71, 1301-1308.

Official and Tentative Methods and Recommended Practices of the American Oil Chemists Society. 3 th Edition, edited by D. Firestone, AOCS, Champaign, IL, USA. (1993) Methods: Ca 5a-40, Cd 8-53 and Cd 20-91.

Sebedio, J. L.; Septier, CH., Grandgirard, A. (1986). - «Fractionation of commercial frying oil samples using Sep-Park cartridges".- J. Am. Oil Chem. Soc. 63, 1541. 1543.

Sebedio, J. L., Astorg, P. O., Septier, C., Grandgirard, A. (1987). - "Quantitative analyses of polar components in frying oils by the latroscan Thin-layer Chromatography Flame Ionization Detection technique".- J. Chromatogr. 405, 371-378.

Shahidi, F. (1995). - «Stability of fats and oils».- In Congreso y Exposición Latinoamericano sobre Processamiento de Grasas y Aceites, Editores. D. Barrera-Arellano, M. A. B. Regitano d'Arce y L. A. G. Gonçalves 6, 47-54. São Paulo, $\mathrm{Br}$.

Standard Methods for Analysis of Oils, Fats and Derivatives. - In, IUPAC App. Chem. Commission on Oils, Fats and Derivatives.- 7 th edition, edited by $C$. Paquot and A. Hautfenne, Blackwell Scientific Publications, Oxford, England. (1987) Method: 2.507.

Taylor, S. L., Berg, C. M., Shoptaugh, N. H., Traisman, E. (1983). - «Mutagen formation in deep-fat fried foods as a function of frying conditions".- J. Am. Oil Chem. Soc. 60, 576-580.

Warner, K., Frankel, E. N. (1985). - «Flavor stability of soybean oil based on induction periods for the formation of volatile compounds by gas chromatography".- J. Am. Oil Chem. Soc. 62, 100-103.

White, P. J. (1991). - «Methods for measuring changes in deep-fat frying oils».- Food Technol. 45, 75-80.

Recibido: Noviembre 1996 Aceptado: Mayo 1997 